\title{
O NÍVEL DE ATIVIDADE FÍSICA INFLUENCIA O CONTROLE POSTURAL REATIVO APÓS PERTURBAÇÃO POSTURAL EXTERNA INESPERADA EM PACIENTES COM DOENÇA DE PARKINSON.
}

Victor Spiandor Beretta ${ }^{1}$, Paulo Cezar Rocha dos Santos, Marcelo Pinto Pereira, Diego Orcioli Silva, Diego Alejandro Rojas Jaimes, Vinicius Cavassano Zampier, Lilian Teresa Bucken Gobbi

${ }^{1}$ Universidade Estadual Paulista (Unesp), Instituto de Biociências, Departamento de Educação Física, Laboratório de Estudos da Postura e da Locomoção (LEPLO), Rio Claro - SP. E-mail: victor beretta@hotmail.com

\section{RESUMO}

O objetivo foi comparar o controle postural reativo após perturbação externa inesperada entre pacientes com doença de Parkinson (DP) fisicamente ativos e inativos. Participaram do estudo 17 pacientes com DP fisicamente ativos (DPA) e 17 fisicamente inativos (DPI). A perturbação da postura foi realizada pelo deslocamento da base de suporte no sentindo posterior. O controle postural reativo foi analisado por meio dos parâmetros eletromiográficos e do centro de pressão (CoP). O teste t de Student para amostras independentes indicou que o DPI apresentou maior tempo para atingir o pico no músculo Gastrocnêmio Medial e para recuperar a posição estável, maior coativação nos músculos Gastrocnêmio Medial/ Tibial anterior e maior amplitude do deslocamento do CoP em relação ao DPA. A partir dos resultados, podemos concluir que pacientes com DP fisicamente ativos demonstram respostas posturais mais rápidas e adequadas após perturbação externa inesperada quando comparados aos fisicamente inativos, sendo um fator importante no controle postural.

Palavras-chave: Desordens do movimento, ajuste postural reativo, atividade física, perturbação inesperada, instabilidade postural.

\section{THE LEVEL OF PHYSICAL ACTIVITY INFLUENCES THE REACTIVE POSTURAL CONTROL AFTER UNEXPECTED EXTERNAL POSTURAL PERTURBATION IN PATIENTS WITH PARKINSON'S DISEASE.}

\begin{abstract}
The aim was to compare the reactive postural control after unexpected external perturbation in patients with Parkinson's disease (PD) physically active and inactive. Participated 17 physically active (APD) and 17 physically inactive (IPD) PD patients. The perturbation occurred by the posterior displacement of the support base. Postural control was analyzed by the electromyography and of the center of pressure (CoP) parameters. Student's $t$ test for independent samples indicated that IPD showed higher time to peak of the Medial Gatrocnemius muscle and to recover the stable position, greater co-activation in Medial Gatrocnemius /Tibialis Anterior muscles and higher range of CoP displacement in relation to APD. From the results, we can conclude that physically active PD patients demonstrate faster and more adequate postural responses after unexpected external perturbation when compared to physically inactive patients, being an important factor in postural control.
\end{abstract}

Keywords: Movement disorders, reactive postural adjustments, physical activity, unexpected perturbation, postural instability.

\section{INTRODUÇÃO}

Com o processo do envelhecimento ocorrem declínios sensoriais e motores, diminuição da força e declínio cognitivo que são relacionados com o controle postural ${ }^{1-4}$. Como consequência, estes indivíduos têm aumento da incidência de quedas devido à incapacidade de recuperação após a perda de equilíbrio ou após uma perturbação externa da postura ${ }^{2,5,6}$. Estas alterações são mais evidentes na presença de doenças neurodegenerativas, como a doença de Parkinson (DP), podendo ser três vezes maior o 
número de quedas nesta população em relação aos idosos neurologicamente sadios ${ }^{6-8}$. Na DP ocorre a degeneração progressiva dos neurônios da substância negra dos núcleos da base, que entre outras funções regula a atividade muscular e o controle adequado do centro de pressão (CoP) para as respostas posturais ${ }^{9,10}$. Portanto sua degeneração leva a uma dificuldade do controle da postura, ${ }^{7,11}$. Déficits no controle postural, como o aumento da oscilação do CoP e pior resposta de estabilidade após perturbação da postura, são relacionados com o número de quedas em pacientes com $\mathrm{DP}^{10,12-16}$.

As perturbações posturais estão presentes nas atividades da vida diária como, por exemplo, durante a freada de um veículo em movimento, como um ônibus ${ }^{17}$. Com isso, os ajustes posturais reativos se tornam extremamente necessários para a manutenção do equilíbrio e, devem ser flexíveis ${ }^{12}$, ativando diferentes músculos para contrabalancear as forças geradas pela perturbação. O controle postural é uma habilidade motora complexa que tem por base a interação de processos sensóriosmotores $^{3}$. Para a manutenção do equilíbrio o sistema nervoso central (SNC) gera padrões de atividade muscular para regular o centro de massa (CoM) dentro dos limites de estabilidade da base de suporte ${ }^{5,9,18}$, sendo este relacionado com a oscilação do CoP dentro da base de suporte. O CoP pode ser entendido como o ponto de distribuição da força sobre a superfície de suporte $^{19}$, sendo que quanto maior for o deslocamento, amplitude e velocidade média do CoP sem que haja desequilíbrio, maior será a estabilidade do indivíduo ${ }^{10}$. Diversos estudos analisaram o controle postural e suas alterações por meio da atividade muscular e do comportamento do CoM e do $\mathrm{CoP}^{10,20-25}$. A atividade muscular é estudada por meio da eletromiografia (EMG), que é uma técnica que analisa a atividade elétrica (potencial de ação) da tensão muscular ${ }^{26,27}$. Pacientes com DP apresentam excessiva coativação e menores magnitudes de ativação de curta duração, atingindo a força máxima de forma mais lenta ${ }^{20-23}$ nas respostas reativas após perturbação externa. O declínio na capacidade de controlar a postura está relacionado com o envelhecimento e com a $\mathrm{DP}^{3,12}$, e outros fatores como a falta de prática regular de exercícios físicos parece influenciar negativamente o controle postura ${ }^{28}$.
Idosos fisicamente ativos apresentam menor tempo para reagir após um estímulo, demonstrando respostas mais rápidas, o que sugere ser um fator positivo para evitar a ocorrência de quedas nessa população em situações inesperadas ${ }^{29,30}$. Além disso, idosos neurologicamente sadios praticantes de exercícios sensórios-motores apresentam menor latência e melhor resposta postural após perturbação externa ${ }^{31,32}$. Mais especificamente para a DP, a prática de exercícios físicos proporciona melhora do controle postural ${ }^{33-36}$, destacando-se como uma estratégia efetiva para a melhora da qualidade de vida, visto que a instabilidade postural é pouco responsiva ao tratamento farmacológico ${ }^{37}$. Porém, o efeito do nível de atividade física no controle postural após perturbação externa inesperada em pacientes com DP é pouco conhecido. A partir desse contexto, o objetivo deste estudo foi comparar o controle postural reativo após perturbação externa inesperada entre pacientes com DP fisicamente ativos e inativos. Nossas hipóteses são que os pacientes com DP fisicamente ativos demonstram melhor controle postural após perturbação inesperada externa, apresentando menor oscilação do CoP e menores valores nos parâmetros temporais e nas variáveis de amplitude da EMG quando comparados aos fisicamente inativos.

\section{METODOLOGIA}

\section{Sujeitos}

Todos os procedimentos experimentais foram realizados nas dependências do Laboratório de Estudos da Postura e da Locomoção (LEPLO) do Departamento de Educação Física da Universidade Estadual Paulista "Júlio de Mesquita Filho", campus de Rio Claro. O estudo foi aprovado pelo comitê de Ética em Pesquisa da mesma Universidade (CAAE: 52534316.1.0000.5465), e os indivíduos permitiram a participação do estudo após assinatura do Termo de Consentimento Livre e Esclarecido. Participaram desse estudo 34 pacientes com DP, classificados como ativos e inativos de acordo com pontuação obtida no Questionário de Baecke Modificado para Idosos $^{38,39}$. Os critérios de exclusão estabelecidos foram: (i) indivíduos com DP com estágio superior a 3 na escala de Hoehn e Yahr (devido a restrições motoras incapacitantes); (ii) declínio cognitivo (abaixo de 24 pontos no Mini Exame do 
Estado Mental ${ }^{40,41}$; (iii) uso de medicamento que cause efeitos colaterais no equilíbrio e; (iv) histórico de problemas de ordem ortopédica e de visão que impossibilitassem o cumprimento do protocolo experimental. As avaliações clínica e do controle postural dos idosos com DP foram realizadas em estado "ON" (45 a 60 minutos após a ingestão) da medicação específica da doença ${ }^{42,43}$.

\section{Determinação do nível de atividade física}

Para determinação do nível de atividade física e classificação dos indivíduos em ativos e inativos, os pacientes responderam o Questionário de Baecke Modificado para Idosos. Para a pontuação do questionário é realizada a somatória dos cálculos das três áreas (atividades domésticas, atividades esportivas e atividades do lazer) avaliadas neste questionário. Com base no escore, os pacientes com DP foram distribuídos em dois grupos: 17 pacientes com DP fisicamente ativos (DPA - escores $\geq 5$ ) e 17 pacientes com DP fisicamente inativos (DPI - escores $\leq 4)^{44}$. Os indivíduos com DP tiveram o diagnóstico realizado por um neurologista particular e a confirmação do diagnóstico baseado nos critérios do Banco de Cérebro de Londres ${ }^{45}$.

Avaliação clínica

Os pacientes com DP foram clinicamente avaliados por um avaliador experiente por meio da Unified Parkinson's Disease Rating Scale (UPDRS) $^{46}$ para identificar o grau de acometimento da DP. A escala de Hoehn e Yahr ${ }^{47}$ versão adaptada ${ }^{48}$ foi utilizada para identificar o estágio evolutivo da doença. O Mini Exame do Estado Mental (MEEM) ${ }^{43}$ foi utilizado para análise da condição cognitiva dos participantes de ambos os grupos (Tabela1).

\section{Avaliação do controle postural}

Para o controle postural, os indivíduos permaneceram em posição bipodal sobre uma plataforma de força posicionada acima do equipamento RC - SLIDE, utilizado para a perturbação postural ${ }^{49}$. A intensidade da perturbação foi de $15 \mathrm{~cm} / \mathrm{s}$ de velocidade, e deslocamento de $5 \mathrm{~cm}^{49-52}$. O participante sofreu perturbações posturais causadas na base de suporte pela translação da plataforma no sentido posterior de forma inesperada. A perturbação foi considerada inesperada porque o participante foi instruído a subir na plataforma e não foi informado sobre qual seria o teste. A tentativa teve duração total de 20 segundos e a intensidade de deslocamento da perturbação foram as mesmas para todos os participantes ${ }^{49}$. Para garantir a segurança, os indivíduos utilizaram, durante a avaliação do controle postural, um equipamento Arnês fixado a um sistema de polias preso no teto ${ }^{49}$.

Procedimentos experimentais

Um eletromiógrafo com 16 canais (Trigno $^{\mathrm{TM}}$ Wireless System - Delsys, Inc.) com frequência de $2000 \mathrm{~Hz}$ e uma plataforma de força (AccuGait, Advanced Mechanical Technologies, Boston, MA) de $50 \times 50 \mathrm{~cm}$ com frequência de coleta de $200 \mathrm{~Hz}$ foram utilizados para aquisição da atividade eletromiográfica e do CoP, respectivamente. Sensores Trigno (eletrodos ativos) foram posicionados nos músculos: bíceps femoral (BF), vasto medial (VM), gastrocnêmio medial (GM) e tibial anterior (TA) do membro mais afetado para os pacientes com $\mathrm{DP}^{49}$. $\mathrm{O}$ membro mais afetado foi determinado por meio dos itens 20-26 da parte motora da UPDRS $^{24,25,53,54}$. Estes músculos foram escolhidos por serem os mais ativados para a manutenção do equilíbrio nessa direção ${ }^{55}$ e na reação a uma perturbação ${ }^{56}$. Os procedimentos para colocação dos foram seguindo as recomendações da SENIAM ${ }^{57}$.

Análise de dados dos dados

Os dados de EMG e do CoP foram coletados por um período de $2000 \mathrm{~ms}$ antes da perturbação até o final da tentativa (20000 ms). O controle postural reativo foi analisado por um período de janelamento entre o início da atividade muscular após a perturbação e 700 $\mathrm{ms}^{49}$, para a EMG (Figura 1). Já para os parâmetros do CoP no controle postural reativo foi realizado um período de janelamento entre o início da perturbação e $700 \mathrm{~ms}^{49}$ (Figura 2). 0 período de repouso foi determinado por um janelamento entre $500 \mathrm{~ms}$ e $300 \mathrm{~ms}$ antes da perturbação $0^{10,21-23,58,59}$. 


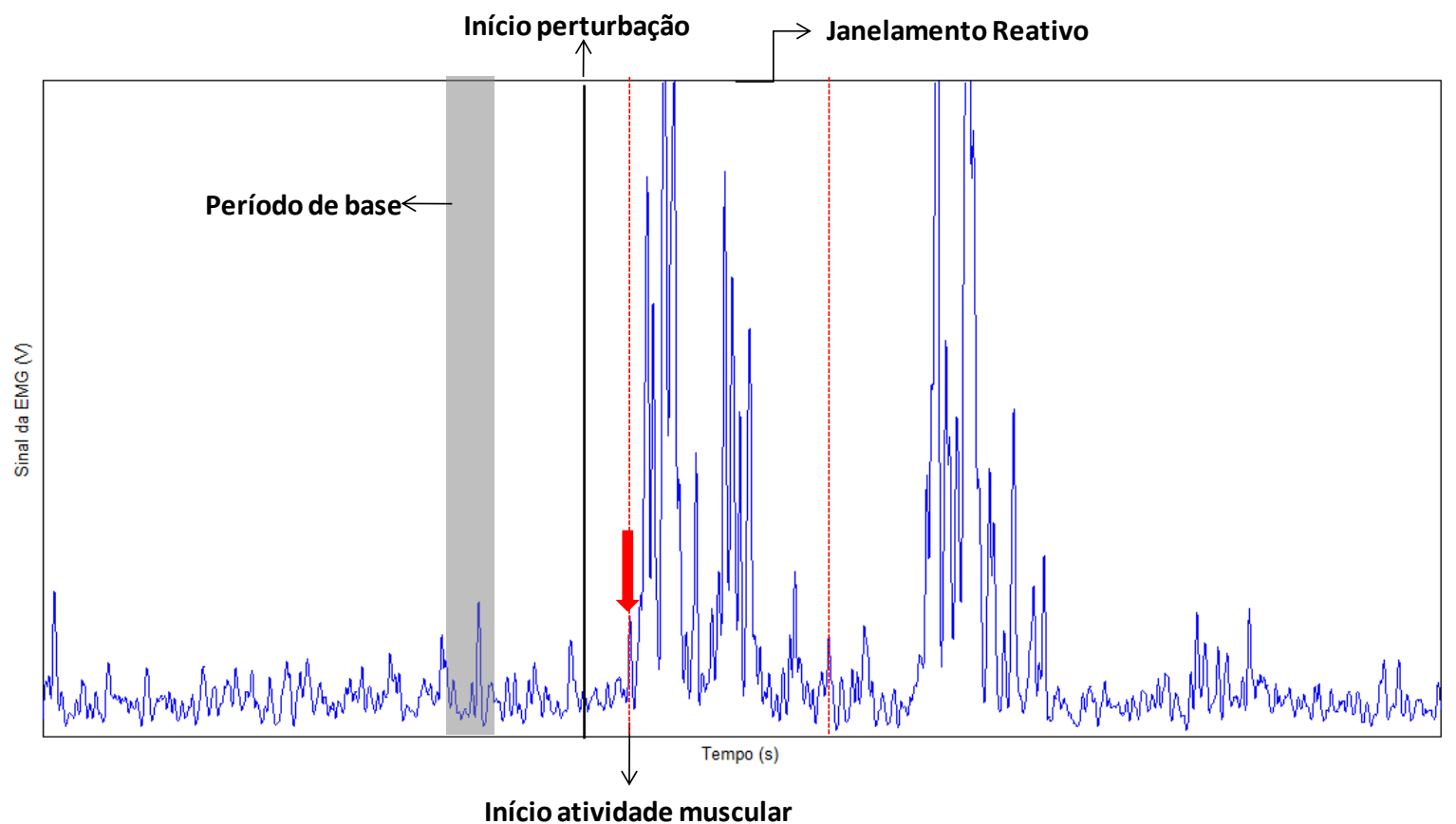

Figura 1. Pontos de interesse na análise da atividade muscular no controle postural reativo após a perturbação inesperada por meio do sinal eletromiográfico.

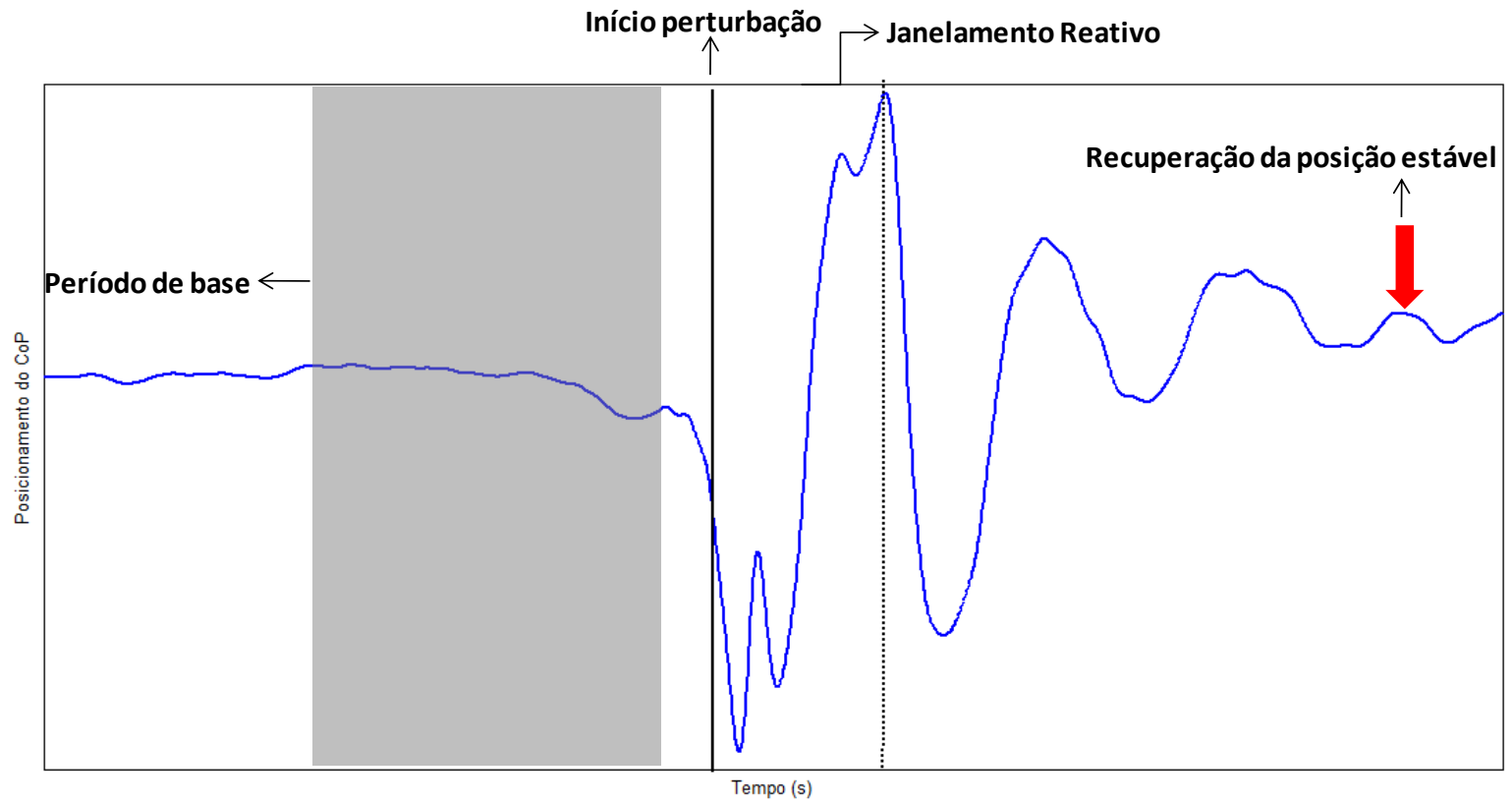

Figura 2. Pontos de interesse na análise do CoP no controle postural reativo após a perturbação inesperada.

Análise dos parâmetros EMG

O sinal de EMG foi tratado com um filtro Butterworth de 4 a ordem com uma frequência de corte de $20-450 \mathrm{~Hz}$ (filtro passa-banda) e após a retificação os dados foram novamente tratados por um filtro Butterworth de $2^{a}$ ordem com uma frequência de corte de $50 \mathrm{~Hz}$ (passa-baixa) ${ }^{49}$. Além disso, o sistema conta com taxa de rejeição de $80 \mathrm{db}$ e a resolução da placa $D$ é de 16 bits. $A$ sincronização do início da perturbação com a
EMG foi realizada por meio de um acelerômetro (Trigno $^{\text {TM }}$ Wireless System - Delsys, Inc.) com frequência de $148,15 \mathrm{~Hz}$ posicionado na plataforma de força ${ }^{49}$. Para o período de base (utilizado para determinar o início da perturbação) foi analisada a atividade do acelerômetro por um período $2000 \mathrm{~ms}$ na atividade estável do acelerômetro antes da perturbação. $O$ início da perturbação foi determinado por um algoritmo semi-automático 
no momento em que a aceleração foi maior que a média +2 desvios padrão do período de base ${ }^{49}$.

O início da atividade muscular foi determinado por um algoritmo semi-automático no momento em que o seu valor foi maior que a média +2 desvios padrão do período de base por pelo menos $30 \mathrm{~ms}^{60}$. O tempo de latência (intervalo de tempo entre início da perturbação e início da atividade muscular) e o tempo para atingir o pico (intervalo entre o início da atividade muscular e o pico da ativação) foram analisados $^{49,59,60}$. Além disso, foram analisadas a magnitude da ativação muscular (iEMG) (calculada pela área embaixo da curva) e a coativação muscular. A iEMG de cada janelamento foi normalizada pela iEMG do períodos de base $\left(100+\left(100 *\right.\right.$ (ilEMG $_{\text {janelamento }}{ }^{-}$ $\left.\left.\left.\left(\mathrm{iEG}_{\text {base }}\right) / \mathrm{iEMG}_{\text {base }}\right)\right)\right)^{21,49,59}$. Ainda, após a normalização, a coativação dos pares BF/VM e GM/TA foi determinada por meio da razão $\left(\left(\mathrm{EMG}_{\text {antagonista }} / \mathrm{iEMG}_{\text {agonista }}\right) * 100\right)^{49,59}$.

\section{Análise dos dados cinéticos}

O sinal do CoP foi tratado por um filtro Butterworth de 4a ordem com uma frequência de corte de $50 \mathrm{~Hz}$ (passa-baixa). Os parâmetros do CoP analisados no sentido anteroposterior foram: amplitude de deslocamento, deslocamento e velocidade média ${ }^{19,61}$. Além disso, por meio do CoP, foi analisado o tempo de recuperação para aposição estável ${ }^{49}$. Para as variáveis do CoP foi determinado $o$ início da perturbação no momento que o deslocamento do CoP foi maior que a média +2 desvios padrão referente à média do valor da linha de base (mesma forma que a descrita para o início da perturbação por meio do acelerômetro) ${ }^{49}$.

Para análise estatística, o nível de significância foi considerado em 0,05 para todas as análises. Os testes de Shapiro-Wilk e de Levene foram empregados para verificação da normalidade na distribuição dos dados e da homogeneidade das variâncias, respectivamente. Os dados de caracterização dos grupos e análise das diferenças entre os grupos no controle postural foram comparados por meio do teste $t$ de Student para amostras independentes.

\section{RESULTADOS}

A Tabela 1 apresenta os dados clínicos, cognitivos e antropométricos dos idosos com DP fisicamente ativos e inativos. A estatística indicou que os DPA apresentaram melhor condição cognitiva quando comparados aos DPI $(p=0,037)$.

Tabela 1. Características antropométricas, clínicas e cognitivas dos idosos com DP fisicamente ativos (DPA) e inativos (DPI).

\begin{tabular}{lccc}
\hline & DPA & DPI & p \\
\hline Idade (anos) & $69,12 \pm 8,21$ & $72,82 \pm 7,78$ & 0,186 \\
Peso $(\mathrm{kg})$ & $69,59 \pm 11,04$ & $70,05 \pm 11,19$ & 0,906 \\
Altura (cm) & $163,66 \pm 10,04$ & $161,58 \pm 8,94$ & 0,526 \\
UPDRS III (pts) & $22,53 \pm 8,92$ & $27,12 \pm 11,17$ & 0,195 \\
UPDRS total (pts) & $34,82 \pm 12,21$ & $44,41 \pm 15,27$ & 0,052 \\
H\&Y (estágio) & $1,94 \pm 0,30$ & $2,15 \pm 0,42$ & 0,112 \\
MEEM (pts) & $28,53 \pm 1,28$ & $27,38 \pm 1,75$ & $0,037 *$
\end{tabular}

UPDRS = Unified Parkinson's Disease Rating Scale; H\&Y = Hoehn e Yahr; MEEM = Mini Exame do Estado Mental. * indica diferença significativa entre os grupos DPA e DPI.

$\mathrm{O}$ teste $\mathrm{t}$ de Student para amostras independentes revelou que os idosos fisicamente inativos apresentaram maior tempo para atingir o pico no músculo $G M\left(t_{32}=-2,575, p=0,015\right)$ (Figura 3a) e maior tempo para recuperar a posição estável $\left(\mathrm{t}_{31}=-2,473, \mathrm{p}=0,019\right)$ quando comparados aos idosos com DP fisicamente ativos (Figura 3b). Ainda, a estatística indicou que os idosos fisicamente inativos apresentaram maior coativação nos músculos GM/TA ( $t_{31}=$ $2,083, p=0,046$ ) (Figura 3c) e maior amplitude do deslocamento do $\operatorname{CoP}\left(t_{31}=-2,470, p=0,019\right)$ em relação aos idosos com DP fisicamente ativos (Figura $3 \mathrm{~d}$ ). Além disso, a análise estatística não revelou diferença significativa para as demais variáveis da EMG e do CoP (Tabela 2). 

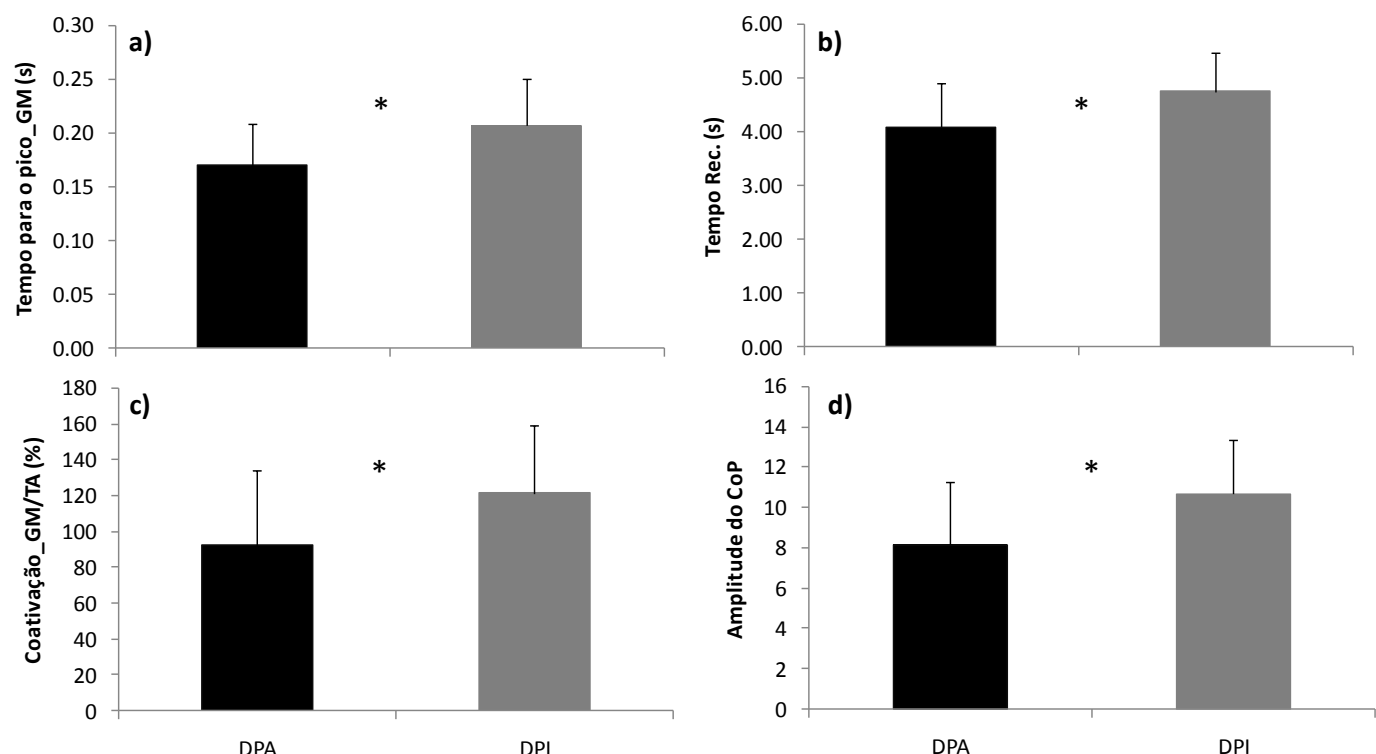

Figura 3. Variáveis da EMG e do CoP que apresentaram diferenças significativas de ambos os grupos: a) tempo para o pico no músculo $\mathrm{GM}$; b) tempo para recuperar a posição estável; c) coativação dos músculos GM/TA; d) Amplitude do deslocamento do CoP. ${ }^{*}$ indica diferença significativa entre os grupos DPA e DPI. DPA = Doença de Parkinson Ativos; DPI = Doença de Parkinson Inativos; Rec. = recuperação.

Tabela 3 2. Variáveis espaciais (EMG e CoP) que não apresentaram diferença significativa entre os grupos.

\begin{tabular}{lccc}
\hline Variáveis & DPA & DPI & p \\
\hline EMG & & & \\
Lat_BF (s) & $0,21 \pm 0,07$ & $0,24 \pm 0,08$ & 0,390 \\
Lat_VM (s) & $0,21 \pm 0,07$ & $0,26 \pm 0,10$ & 0,078 \\
Lat_GM (s) & $0,16 \pm 0,04$ & $0,18 \pm 0,03$ & 0,189 \\
Lat_TA (s) & $0,22 \pm 0,08$ & $0,28 \pm 0,12$ & 0,092 \\
TTP_BF (s) & $0,19 \pm 0,19$ & $0,28 \pm 0,17$ & 0,160 \\
TTP_VM (s) & $0,28 \pm 0,23$ & $0,22 \pm 0,23$ & 0,492 \\
TTP_TA (s) & $0,45 \pm 0,23$ & $0,34 \pm 0,30$ & 0,251 \\
iEMG_BF (\% do período base) & $690,02 \pm 731,27$ & $389,94 \pm 213,08$ & 0,114 \\
iEMG_VM (\% do período base) & $520,58 \pm 605,09$ & $429,11 \pm 234,96$ & 0,565 \\
iEMG_GM (\% do período base) & $444,19 \pm 318,47$ & $581,50 \pm 501,74$ & 0,359 \\
iEMG_TA (\% do período base) & $542,81 \pm 473,11$ & $633,16 \pm 501,38$ & 0,607 \\
Coativação BF/VM (\%) & $86,51 \pm 49,93$ & $112,54 \pm 55,03$ & 0,158 \\
CoP & & & 0,592 \\
Deslocamento (cm) & $12,85 \pm 4,49$ & $27,45 \pm 12,19$ & 0,592 \\
Velocidade média (cm/s) & $25,45 \pm 8,89$ & &
\end{tabular}

DPA = DP ativos; DPI = DP inativos; Lat = latência; TTP = tempo para o pico; iEMG = integral EMG.

Colloq Vitae2017 set-dez; 9(3): 48-58. DOI: 10.5747/cv.2017.v09.n3.v209

ISSN 1984-6436/C 2017 - Publicado pela Universidade do Oeste Paulista.

Artigo Open Access sob uma licença CC BY-NC-ND (http://creativecommons.org/licenses/by-nc-nd/4.0/). 


\section{DISCUSSÃO}

O presente estudo teve como objetivo comparar o controle postural reativo após perturbação externa inesperada entre pacientes com doença de Parkinson fisicamente ativos e inativos. A partir dos resultados encontrados, nossas hipóteses foram parcialmente confirmadas: idosos com DP fisicamente ativos apresentaram melhor condição cognitiva e melhor controle postural reativo, evidenciado pelo menor tempo para atingir o pico da atividade muscular do GM e menor tempo para recuperar a posição estável. Além disso, os idosos com DP fisicamente ativos apresentaram menor coativação nos músculos $\mathrm{GM} / \mathrm{TA}$ e menor amplitude do deslocamento do CoP quando comparados aos fisicamente inativos. Porém, de maneira inesperada, não foi encontrada diferença significativa entre os grupos no tempo de latência, principalmente dos músculos agonistas (GM e BF).

O declínio do controle postural em idosos pode ser devido aos déficits na habilidade do sistema na recuperação do equilíbrio após uma perturbação ou pelo déficit da na capacidade de conter a perturbação ${ }^{62}$. A perturbação externa inesperada pode ocorrer nas atividades da vida diária e podem ser associadas com o aumento da ocorrência de quedas ${ }^{63}$, necessitando de respostas musculares adequadas para controlar a postura ${ }^{64}$. Pacientes com DP apresentam alteração na atividade muscular e na coordenação muscular após perturbação externa ${ }^{12,20-23,56}$. A ativação muscular descoordenada parece aumentar a rigidez destes pacientes, o que dificulta ainda mais a manutenção do equilíbrio ${ }^{21,22}$.

No controle postural reativo as respostas musculares rápidas e coordenadas ${ }^{21,22}$ parecem diminuir a oscilação e a amplitude do deslocamento do $\mathrm{CoM}$ e do $\mathrm{CoP}^{10,12}$. Alguns estudos apontaram que a prática regular de atividade física em idosos neurologicamente sadios propiciam benefícios para os componentes sensórios-motores, melhoram o tempo de latência e diminuem o desequilíbrio na postura após perturbação externa em idosos neurologicamente sadios $^{31,32}$. A diminuição no tempo de latência indica que 0 indivíduo respondeu de forma mais rápida após a perturbação ${ }^{59}$. Porém, em nosso estudo não foi evidenciado este resultado em idosos com DP fisicamente ativos. Além dos efeitos positivos em idosos neurologicamente sadios, a atividade física promove melhora no equilíbrio em pacientes com $\mathrm{DP}^{33,65}$. O exercício físico parece ser capaz de estimular a síntese de dopamina nas células dopaminérgicas presentes e a inatividade física está relacionada com o declínio no desempenho das atividades da vida diária ${ }^{66}$. Além disso, a inatividade física parece acelerar o processo de envelhecimento nessa população e com os consequentes acometimentos da senescência ${ }^{67}$.

Apesar de não encontradas diferenças no tempo de latência, os idosos com DP fisicamente ativos apresentam respostas mais rápidas após a ocorrência de perturbação externa inesperada, podendo ser observada pelo menor tempo para atingir o pico da atividade do músculo agonista nessa condição (GM) e pelo menor tempo para recuperar a posição estável. Esses resultados indicam efeitos positivos do nível de atividade física na resposta postural, sendo que esses pacientes ${ }^{58}$ demonstraram ativação em nível máximo de forma mais rápida ${ }^{59}$ e permaneceram menos tempo em situação de desequilíbrio. Além disso, os idosos com DP fisicamente ativos demonstraram resposta mais adequada à perturbação, apresentando menor amplitude do deslocamento do CoP e menor coativação dos músculos GM/TA. Estes podem ser indicadores de um menor risco de ocorrência de quedas nos pacientes ${ }^{63}$, representado grande relevância, uma vez que as respostas inadequadas, como citadas nos parágrafos anteriores, indicam déficit no controle postural nessa população ${ }^{10,12,21,22,68}$.

Evidências têm apontado que a prática regular de atividade física é benéfica para a manutenção da postura ${ }^{33,35}$. Programas de exercícios com o foco na exposição dos indivíduos às perturbações demonstraram efeitos positivos no controle postural, podendo ser eficiente para a diminuição da ocorrência de quedas em idosos neurologicamente sadios e com $\mathrm{DP}^{17,35}$. Além disso, o estudo realizado por Gobbi et al. ${ }^{33}$ indicou que a prática de exercícios multimodais e exercícios mais gerais com o foco em alterar os efeitos da inatividade física (melhora da força, flexibilidade e coordenação motora) são capazes de proporcionar melhora no equilíbrio em idosos com DP em tarefas sem a presença de perturbação externa. Estes resultados sugerem que a prática da atividade física é uma forma de tratamento importante para o controle postural 
na DP. Além dos benefícios para o controle postural de idosos com DP, cabe destacar que o nível de atividade física parece ser um importante fator para a melhora na condição cognitiva desses pacientes ${ }^{69-71}$.

Apesar de importantes achados, o presente estudo apresentou algumas limitações como a análise do controle postural apenas na perturbação externa inesperada e a falta de grupos com idosos neurologicamente sadios fisicamente ativos e inativos para a comparação com as respostas dos pacientes com DP. Sugerimos que próximos estudos analisem 0 efeito do nível de atividade física na habituação do controle postural à perturbação externa. Além disso, há a necessidade de estudos futuros analisarem o efeito de diferentes programas de exercício físico no controle postural reativo em pacientes com DP.

\section{CONCLUSÃO}

A partir dos nossos resultados é possível concluir que o nível de atividade física é um importante fator para o controle postural em pacientes com DP após uma perturbação externa inesperada. Idosos com DP fisicamente ativos demonstraram respostas posturais mais rápidas (menor tempo para atingir o pico e para recuperar a posição estável) e mais adequadas (menor coativação e amplitude do CoP) quando comparados aos idosos fisicamente inativos, sugerindo melhor controle postural nesses indivíduos.

\section{AGRADECIMENTOS}

Os autores agradecem à Fundação de Amparo à Pesquisa do Estado de São Paulo (FAPESP) [processo no 2016/00503-0] pelo suporte financeiro.

\section{CONFLITO DE INTERESSE}

Os autores declaram não haver qualquer potencial conflito de interesse que possa interferir na imparcialidade deste trabalho científico.

\section{REFERÊNCIAS}

1. Lord SR, Ward JA, Williams P, Anstey J. Physiological factors associated with falls in older communitydwelling women. J Am Geriat Soc. 1994;42:1110-7. DOI: https://doi.org/10.1111/i.1532-
2. Tinetti ME, Inouye SK, Gill TM, Doucette JT. Shared risk factors for falls, incontinence and functional dependence: unifying the approach to geriatric syndromes. JAMA. 1995;273(17):1348-53. DOI: http://dx.doi.org/10.1001/jama.1995.0352041004202 $\underline{4}$

3. Horak FB. Postural orientation and equilibrium: what do we need to know about neural control of balance to prevent falls? Age Ageing. 2006;35(2):ii7ii11. DOI: https://doi.org/10.1093/ageing/afl077

4. Lirani-Silva E, Beretta VS, Jimenez AMF, Gobbi, LTB. Postural control and somatosensory information: effects of aging and Parkinson's disease. In: Barbieri FA, Vitório R. (Eds.). Locomotion and posture in older adults: The role of aging and movement disorders. Springer International Publishing, 2017. p.307-22. DOI: https://doi.org/10.1007/978-3-319-48980-3 20

5. Maki BE, Mcilroy WE. Postural control in the older adult. Clin Geriatr Med. 1996;12(4):635-58.

6. Christofoletti G, Oliani MM, Gobbi LTB, Gobbi S, Stella F. Risco de quedas em idosos com doença de Parkinson e demência de Alzheimer: um estudo transversal. Rev Bras Fisioter. 2006; 10(4):429-33. DOI: https://doi.org/10.1590/S1413-35552006000400011

7. Bloem BR, Grimbergen YA, Cramer $M$, Willemsen $M, Z$ winderman $A H$. Prospective assessment of falls in Parkinson's disease. J Neurol. 2001;248(11):950-8. DOI: https://doi.org/10.1007/s004150170047

8. Pickering RM, Grimbergen YA, Rigney U, Ashburn A, Mazibrada G, Wood B, et al. A meta-analysis of six prospective studies of falling in Parkinson's disease. Mov Disord. 2007;15;22(13): 1892-900. https://doi.org/10.1002/mds.21598

9. Horak FB, Macpherson JM. Postural orientation and equilibrium. In: Rowell LB, Shepard JT (Eds.). Handbook of physiology - section 12. Exercise: regulation and integration of multiple system. New York, Oxford University Press, 1996, p.255-92.

10. Horak FB, Dimitrova D, Nutt JG. Direction-specific postural instability in subjects with Parkinson's disease. Exp Neurol. 2005;193(2):504-21. DOI: https://doi.org/10.1016/i.expneurol.2004.12.008

11. Wichmann T, DeLong MR. Núcleos da Base. In Kandel ER, Schwarts JH, Jessell TM, Siegelbaum SA, Hudspeth AJ. Princípios de neurociências. 5.ed. Porto Alegre: AMGH, 2014. p.811-32.

12. Horak FB, Nutt JG, Nashner LM. Postural inflexibility in parkinsonian subjects. J Neurol Sci. 1992;111(1):46-58. DOI: https://doi.org/10.1016/0022-510X(92)90111-W

5415.1994.tb06218.x 
13. Ashburn A, Stack E, Pickering RM, Ward CD. A community-dwelling sample of people with Parkinson's disease: characteristics of fallers and nonfallers. Age Ageing. 2001;30(1):47-52. DOI: https://doi.org/10.1093/ageing/30.1.47

14. Adkin AL, Frank JS, Jog MS. Fear of falling and postural control in Parkinson's disease. Mov Disord. 2003;18(5):496-502.

DOI:

https://doi.org/10.1002/mds.10396

15. Matinolli $M$, Korpelainen JT, Korpelainen $R$, Sotaniemi KA, Virranniemi M, Myllyla VV. Postural sway and falls in Parkinson's disease: a regression approach. Mov Disord. 2007;22:1927-35. DOI: http://dx.doi.org/10.1002/mds.21633

16. Latt MD, Lord SR, Morris JG, Fung VS. Clinical and physiological assessments for elucidating falls risk in Parkinson's disease. Mov Disord. 2009;24:1280-9. DOI: https://doi.org/10.1002/mds.22561

17. Mansfield A, Wong JS, Bryce J, Knorr S, Patterson KK. Does perturbation-based balance training prevent falls? Systematic review and meta-analysis of preliminary randomized controlled trials. Phys Ther. 2015;95(5):700-9.

https://doi.org/10.2522/pti.20140090

18. Macpherson JM, Horak FB. Postura. In Kandel ER, Schwarts JH, Jessell TM, Siegelbaum SA, Hudspeth AJ. Princípios de neurociências. 5.ed. Porto Alegre: AMGH, 2014. p.811-32.

19. Duarte M, Freitas SMSF. Revisão sobre posturografia baseada em plataforma de força para avaliação do equilíbrio. Rev Bras Fisioter. 2010;14(3):183-92. DOI: https://doi.org/10.1590/S1413-35552010000300003

20. Bloem BR. Postural instability in Parkinson's disease Clin Neurol Neurosurg. 1992;94(Suppl):S41-5. DOI: https://doi.org/10.1016/0303-8467(92)90018-X

21. Henry SM, Fung J, Horak FB. EMG responses to maintain stance during multidirectional surface translations. J Neurophysiol. 1998;80(4):1939-50. DOI: https://doi.org/10.1152/in.1998.80.4.1939

22. Dimitrova D, Horak FB, Nutt JG. Postural muscle responses to multidirectional translations in patients with Parkinson's disease. J Neurophysiol. 2004;91(1):489-501. DOI: https://doi.org/10.1152/jn.00094.2003

23. Dimitrova D, Nutt JG, Horak FB. Abnormal forces patterns for multidirectional postural responses in patients with Parkinson's disease. Exp Brain Res. 2004;156(2):183-95. DOI: https://doi.org/10.1007/s00221-003-1770-4
24. Beretta VS, Gobbi LT, Lirani-Silva E, Simieli L, Orcioli-Silva D, Barbieri FA. Challenging Postural Tasks Increase Asymmetry in Patients with Parkinson's Disease. PLoS One. 2015;10(9): e0137722. DOI: https://doi.org/10.1371/journal.pone.0137722

25. Barbieri FA, Polastri PF, Baptista AM, Lirani-Silva E, Simieli L, Orcioli-Silva D, et al. Effects of disease severity and medication state on postural control asymmetry during challenging postural tasks in individuals with Parkinson's disease. Hum Mov Sci. 2016;46:96-103. DOI: https://doi.org/10.1016/j.humov.2015.12.009

26. Enoka RM. Bases neuromecânicas da cinesiologia. Barueri: Manole; 2000.

27. Winter DA. Kinesiological electromyography. In: Winter DA. Biomechanics and motor control of human movement. 4.ed. New York: John Wiley \& Sons; 2009. p.250-280.

DOI:

https://doi.org/10.1002/9780470549148.ch10

28. Perrin P, Deviterne D, Hugel F, Perrot C. Judo, better than dance, develops sensorimotor adaptabilities involved in balance control. Gait Posture. 2002;15(2):187-94. DOI: https://doi.org/10.1016/S0966-6362(01)00149-7

29. Lord SR, Ward JA, Williams P, Strudwick M. The effect of a 12-month exercise trial on balance, strength, and falls in older women: a randomized controlled trial. J Am Geriatr Soc. 1995;43(11):1198206. DOI: $\quad$ https://doi.org/10.1111/i.15325415.1995.tb07394.x

30. Hunter GR, Wetzstein CJ, McLafferty $\mathrm{CL}$ Jr, Zuckerman PA, Landers KA, Bamman MM. Highresistance versus variable resistance training in older adults. Med Sci Sports Exerc. 2001;33(10):1759-64. DOI: https://doi.org/10.1097/00005768-200110000$\underline{00022}$

31. Granacher U, Gollhofer A, Strass D. Training induced adaptations in characteristics of postural reflexes in elderly men. Gait Posture. 2006;24(4):45966.

DOI:

https://doi.org/10.1016/j.gaitpost.2005.12.007

32. Lopes AG. Efeitos da atividade física no controle postural e capacidade funcional de idosos. [Tese]. Rio Claro: Instituto de Biociências da Universidade Estadual Paulista, 2010.

33. Gobbi LTB, Oliveira-Ferreira MD, Caetano MJ, Lirani-Silva E, Barbieri FA, Stella F, et al. Exercise programs improve mobility and balance in people with Parkinson's disease. Parkinsonism Relat Disord. 2009;15(Suppl 3):S49-52. DOI: https://doi.org/10.1016/S1353-8020(09)70780-1 
34. Nocera J, Horvat M, Ray CT. Effects of home-based exercise on postural control and sensory organization in individuals with Parkinson disease. Parkinsonism Relat Disord. 2009;15(10):742-5. DOI: https://doi.org/10.1016/i.parkreldis.2009.07.002

35. Smania N, Corato $E$, et al. Effect of balance training on postural instability in patients with idiopathic Parkinson's disease. Neurorehabil Neural Repair. 2010;24(9):826-34. DOI: https://doi.org/10.1177/1545968310376057

36. Barbieri FA, Simieli L, Orcioli-Silva D, Gobbi LTB. Benefícios do exercício físico para pacientes com doença de Parkinson. In: Coelho FGM, Gobbi S, Costa, JLR, Gobbi LTB. Exercício físico no envelhecimento saudável e patológico: da teoria à prática. Curitiba: Editora CRV; 2013. 329p.

37. Nonnekes J, Timmer MH, de Vries NM, Rascol $\mathrm{O}$, Helmich RC, Bloem BR. Unmasking levodopa resistance in Parkinson's disease. Mov Disord. 2016;31(11):1602-9. DOI: https://doi.org/10.1002/mds.26712

38. Baecke JA, Burema J, Frijters JE. A short questionnaire for the measurement of habitual physical activity in epidemiological studies. Am J Clin Nutr. 1982;36(5):936-42. DOI: https://doi.org/10.1037/t15903-000

39. Florindo $A A$, Latorre MSDO. Validação e reprodutibilidade do questionário BAECKE de avaliação da atividade física habitual em homens adultos. Rev Bras Med Esporte. 2003;9:121-8.

40. Folstein MF, Folstein SE, McHugh PR. Mini-Mental State: a practical method for grading the cognitive state of patients for clinician. J Psychiatr Res. 1975;12:189-98. DOI: https://doi.org/10.1016/00223956(75)90026-6.

41. Brucki SMD, Nitrini R, Caranelli P, Bertolucci PHF, Okamoto IH. Sugestões para o uso do mini-exame do estado mental no Brasil. Arq Neuro-Psiquiatr. 2003;61(3B):777-81. DOI: https://doi.org/10.1590/S0004-282X2003000500014

42. Carpenter MG, Bloem BR. Postural control in Parkinson patients: a propioceptive problem? Exp Neurol. 2011;227(1):26-30. DOI: https://doi.org/10.1016/j.expneurol.2010.11.007

43. Espay AJ, Fasano A, van Nuenen BF, Payne MM, Snijders AH, Bloem BR. "On" state freezing of gait in Parkinson disease: a paradoxical levodopa-induced complication. Neurology. 2012;78(7):454-7. DOI: https://doi.org/10.1212/WNL.0b013e3182477ec0

44. Santos P, Barbieri, F, Orcioli-Silva D, Simieli L, Gobbi L. Effects of physical activity levels on fatigue perception in patients with pParkinson's disease and neurologically healthy individuals. Health. 2014;6:2927-33. DOI: https://doi.org/10.4236/health.2014.621331.

45. Hughes AJ, Daniel SE, Kilford L, Lees AJ. Accuracy of clinical diagnosis of idiopathic Parkinson's disease: a clinico-pathological study of 100 cases. J Neurol Neurosurg Psychiatry. 1992;55(3):181-4. DOI: https://doi.org/10.1136/innp.55.3.181

46. Fahn $\mathrm{S}$, Elton R. Members of the UPDRS. Development Comitee. The unified Parkinson's disease rating scale. In: Fahn $S$, Marsden CD, Calne DB, Goldstein M. (Eds.). Recent developments in Parkinson's disease, v.2. Florham Park NJ: Mcmellam Health Care Information; 1987. p.153-63.

47. Hoehn MM, Yahr MD. Parkinsonism: onset, progression and mortality. Neurology. 1967;17:57381.

DOI:

http://dx.doi.org/10.1212/WNL.17.5.427:1526-632X

48. Schenkman M, Wei Zhu C, Cutson TM, WhettenGoldstein K. Longitudinal evaluation of economic and physical impact of Parkinson's disease. Parkinsonism Relat Disord. 2001;8(1):41-50. DOI: https://doi.org/10.1016/S1353-8020(00)00079-1

49. Beretta VS. Ajustes posturais sob perturbação externa em indivíduos com doença de Parkinson e neurologicamente sadios [dissertação]. Rio Claro: Instituto de Biociências da Universidade Estadual Paulista, 2017.

50. Chong RK, Horak FB, Woollacott MH. Timedependent influence of sensorimotor set on automatic responses in perturbed stance. Exp Brain Res. 1999;124(4):513-9.

https://doi.org/10.1007/s002210050647

51. Smith BA, Jacobs JV, Horak FB. Effects of magnitude and magnitude predictability of postural perturbations on preparatory cortical activity in older adults with and without Parkinson's Disease. Exp Brain Res. 2012;222(4):455-70. DOI: https://doi.org/10.1007/s00221-012-3232-3

52. Smith BA, Jacobs JV, Horak FB. Effects of amplitude cueing on postural responses and preparatory cortical activity of people with Parkinson disease. J Neurol Phys Ther. 2014;38(4):207-15. DOI: https://doi.org/10.1097/NPT.0000000000000058

53. Uitti RJ, Baba Y, Whaley NR, Wszolek ZK, Putzke JD. Parkinson disease: Handedness predicts asymmetry. Neurology. 2005;64:1925-1930. DOI: https://doi.org/10.1212/01.WNL.0000163993.82388.C $\underline{8}$

54. Boonstra TA, van Vugt JP, van der Kooij $H$, Bloem $B R$. Balance asymmetry in parkinson's disease and its contribution to freezing of gait. PLoS 
One. 2014;9(7):e102493.

DOI:

https://doi.org/10.1371/journal.pone.0102493.

55. $\mathrm{Hu} \mathrm{MH}$, Woollacott $\mathrm{MH}$. Multisensory training of standing balance in older adults: II. Kinematic and electromyographic postural responses. J Gerontol. 1994;49(2):M62-71.

56. Carpenter MG, Allum JH, Honegger F, Adkin AL, Bloem BR. Postural abnormalities to multidirectional stance perturbations in Parkinson's disease. J Neurol Neurosurg Psychiatry. 2004;75(9):1245-54. DOI: https://doi.org/10.1136/innp.2003.021147

57. Hermens HJ, Freriks B, Disselhorst-Klug C, Rau G. et at. Development of recommendations for SEMG sensors and sensor placement procedures. J Electromyogr Kinesiol. 2000;10(5):361-74. DOI: https://doi.org/10.1016/S1050-6411(00)00027-4

58. Santos MJ, Kanekar N, Aruin AS. The role of anticipatory postural adjustments in compensatory control of posture: 1. Electromyographic analysis. J Electromyogr Kinesiol. 2010;20(3):388-97. DOI: https://doi.org/10.1016/i.jelekin.2009.06.006

59. Freitas PB, Knight CA, Barela JA. Postural reactions following forward platform perturbation in young, middle-age, and old adults. J Electromyogr Kinesiol. 2010;20(4):693-700.

https://doi.org/10.1016/i.jelekin.2009.11.009

60. Cleworth TW, Chua R, Inglis JT, Carpenter, MG. Influence of visual height exposure on postural reactions to support surface translations. Gait Posture. 2016;47:96-102.

DOI:

https://doi.org/10.1016/i.gaitpost.2016.04.006

61. Duarte M. Análise estabilográfica da postura ereta humana quasi-estática. [Tese de Livre Docência em Biomecânica]. São Paulo: Escola de Educação Física e Esporte da Universidade de São Paulo, 2000.

62. Stokes IA, Gardner-Morse M. Spinal stiffness increases with axial load: another stabilizing consequence of muscle action. J Electromyogr Kinesiol. 2003;13(4):397-402. DOI: https://doi.org/10.1016/S1050-6411(03)00046-4

63. Visser JE, Carpenter MG, van der Kooij H, Bloem BR. The clinical utility of posturography. Clin Neurophysiol. 2008;119(11):2424-36. DOI: https://doi.org/10.1016/j.clinph.2008.07.220

64. Oude Nijhuis LB, Allum JH, Valls-Solé J, Overeem $\mathrm{S}$, Bloem BR. First trial postural reactions to unexpected balance disturbances: a comparison with the acoustic startle reaction. J Neurophysiol. 2010;104(5):2704-12. DOI: https://doi.org/10.1152/jn.01080.2009
65. Lun V, Pullan N, Labelle N, Adams C, Suchowersky $O$. Comparison of the effects of a self-supervised home exercise program with a physiotherapistsupervised exercise program on the motor symptoms of Parkinson's disease. Mov Disord. 2005;20:971-5. DOI: https://doi.org/10.1002/mds.20475

66. Sutoo D, Akiyama K. Regulation of brain function by exercise. Neurobiol Dis. 2003;13:1-14. DOI: https://doi.org/10.1016/S0969-9961(03)00030-5

67. Glendinning DS, Enoka RM. Motor unit behavior in Parkinson's disease. Phys Ther. 1994;74:61-70. DOI: https://doi.org/10.1093/pti/74.1.61

68. Bloem BR, Beckley DJ, van Dijk JG, Zwinderman $A H$, Remler MP, Roos RA. Influence of dopaminergic medication on automatic postural responses and balance impairment in Parkinson's disease. Mov Disord. 1996;11(5):509-21. DOI: https://doi.org/10.1002/mds.870110506

69. Murray DK, Sacheli MA, Eng JJ, Stoessl AJ. The effects of exercise on cognition in Parkinson's disease: a systematic review. Transl Neurodegener. 2014;3(1):5. DOI: https://doi.org/10.1186/2047-9158-3-5

70. Teixeira-Arroyo C, Rinaldi NM, Batistela RA, Barbieri FA, Vitório R, Gobbi LTB. Exercise and cognitive functions in Parkinson's disease: Gender differences and disease severity. Motriz. 2014; 20(4):461-9. $\quad$ https://doi.org/10.1590/S1980$\underline{65742014000400014}$

71. David FJ, Robichaud JA, et al. Exercise improves cognition in Parkinson's disease: The PRET-PD randomized, clinical trial. Mov Disord. 2015;30(12):1657-63. DOI: https://doi.org/10.1002/mds.26291

Recebido para publicação em 14/08/2017

Revisado em 18/09/2017

Aceito em 27/09/2017 